\title{
AVALIAÇÃO DE GENÓTIPOS DE SOJA EMSEMEADURA DE INVERNO, EM SELVÍRIA, MS: PRODUÇÃO E QUALIDADE FISIOLÓGICA DE SEMENTES ${ }^{1}$
}

\author{
JOSUÉ BISPO DA SILVA², EDSON LAZARINI ${ }^{3}$, MARCO EUSTÁQUIO DE SÁ ${ }^{4}$
}

\begin{abstract}
RESUMO - A semeadura da soja fora do período convencional tem se tornado uma opção para produção de sementes com elevado potencial fisiológico, o que torna necessária a busca por materiais adaptados às novas condições ambientais, fornecendo, assim, maiores subsídios aos agricultores. A pesquisa teve por objetivo identificar genótipos capazes de produzir sementes de alta qualidade no inverno, em Selvíria, MS. As cultivares FT-2000, FT-Inaê, Embrapa 20 (Doko RC), CAC-1, IAC-17, IAC-18, IAC-19, IAC-8-2, FT-101, FT-109, MT/BR 45 (Paiaguás), MT/BR 50 (Parecis), MT/BR 52 (Curió), MT/BR 53 (Tucano), MT/BR 47 (Canário), MT/BR 49 (Pioneira), BRSMT Uirapuru, BR Emgopa 314 (Garça Branca), MG/BR 46 (Conquista), FT-Estrela, FT-Cometa, Dourados, JAB-11, BR 9 (Savana), FT-Abyara, Embrapa 30 (Vale do Rio Doce), Embrapa 9 (Bays), Embrapa 31 (Mina), IAC-16, IAS 5, EMGOPA-304 e IAC-Foscarin 31 foram semeadas em 5/6/1998 e avaliadas quanto às características agronômicas e o potencial fisiológico das sementes. O delineamento experimental utilizado foi blocos ao acaso, com cinco repetições. As cultivares mais adaptadas às condições climáticas da região foram Parecis, Bays, CAC-1, Garça Branca, Paiaguás, Pioneira e IAC-16; a semeadura no inverno deve ser antecipada para início de maio, com suplementação hídrica, uma vez que junho já foi muito tardia.
\end{abstract}

Termos para indexação: Glycine max, potencial fisiológico, cultivares, safrinha.

\section{EVALUATION OF SOYBEAN CULTIVARS IN WINTER SOWING, IN SELVÍRIA, MS, BRAZIL: PRODUCTION AND PHYSIOLOGICALQUALITY OF SEEDS}

\begin{abstract}
The sowing of soybean out of the conventional period is an option for the production of seeds with high physiological quality. On the other hand, this fact makes necessary the search for cultivars adapted to new environmental conditions. Therefore, additional research is needed to supply more information to producers related to the choice of the most suited cultivars with regard to high seed quality. This research was conducted in Selvíria, MS, Brazil in which the cultivars FT-2000, FT-Inaê, Embrapa 20 (Doko RC), CAC-1, IAC-17, IAC-18, IAC-19, IAC-8-2, FT-101, FT-109, MT/ BR 45 (Paiaguás), MT/BR 50 (Parecis), MT/BR 52 (Curió), MT/BR 53 (Tucano), MT/BR 47 (Canário), MT/BR 49 (Pioneira), BRSMT Uirapuru, BR Emgopa 314 (Garça Branca), MG/BR 46 (Conquista), FT-Estrela, FT-Cometa, Dourados, JAB-11, BR 9 (Savana), FT-Abyara, Embrapa 30 (Vale do Rio Doce), Embrapa 9 (Bays), Embrapa 31 (Mina), IAC-16; IAS 5, EMGOPA-304, and IAC-Foscarin 31 were studied. These cultivars were sown on June 5, 1998, and evaluated both for agronomic characteristics and the physiological potential of the seeds. The experimental units were arranged in the field according to a randomized complete block design with five replicates. The cultivars more adapted to the climatic conditions were Parecis, Bays, CAC-1, Garça Branca, Paiaguás, Pioneira, and IAC-16. It was concluded that winter sowing should start at the beginning of May, with water supply, since June was found to be too late.
\end{abstract}

Index terms: Glycine max, physiological potential, cultivars, early sowing

${ }^{1}$ Submetido em: 02/05/2005. Aceito para publicação em: 30/07/2007

${ }^{2}$ Eng $^{\circ}$ Agr $^{\circ}$, Prof. Adjunto, Depto. Ciências Agrárias, Universidade Federal do Acre - UFAC, BR 364, km 04, 69915-900, Rio Branco, AC. End. Eletrônico: jbsilva@fcav.unesp.br

${ }^{3}$ Eng $^{\circ}$ Agr $^{\circ}$, Livre Docente, Departamento de Fitotecnia, Tecnologia de Alimentos e Sócio-Economia, Universidades Estadual Paulista - UNESP, Câmpus de Ilha Solteira, SP. Cx. Postal 31, 15385-000.

${ }^{4}$ Eng $^{\mathrm{o}} \mathrm{Agr}^{\mathrm{o}}$, Professor Titular, Departamento de Fitotecnia, Tecnologia de Alimentos e Sócio-Economia, UNESP, lha Solteira. 


\section{INTRODUÇÃO}

Informações obtidas nas fases iniciais de grande ampliação do plantio de soja mostram que, nos Estados tradicionalmente produtores, a semeadura no mês de novembro tem apresentado os melhores resultados em termos de produtividade (Nakagawa et al., 1983). No entanto, para as culturas implantadas nesse mês, a maturação e a colheita ocorrem em meados de março, época em que freqüentemente se verifica ocorrência de alta pluviosidade e de temperaturas elevadas, fatores ambientais considerados adversos quando se busca a produção de sementes com elevado potencial fisiológico.

Pesquisas a respeito de semeadura fora da época convencional, realizadas tanto em outros países quanto no Brasil, têm mostrado que, para diferentes cultivares, as sementes apresentam, em relação às semeadas em época convencional (outubro e novembro), potencial fisiológico superior na entre-safra, pois nesse período a maturação e a colheita coincidirão com períodos de menores temperatura e umidade (TeKrony et al., 1984; Paolinelli et al., 1984; Tragnago e Bonetti, 1984; Nakagawa et al., 1984 a,b; Medina et al., 1997b; Lazarini et al., 2001; Crusciol et al., 2002), embora nos trabalhos de Braccini et al. (2003) as sementes de cinco cultivares avaliadas apresentassem melhor qualidade fisiológica e sanitária quando a semeadura ocorreu em novembro, em comparação com as semeadas na safrinha (janeiro e fevereiro).

Um aspecto negativo possível é quanto ao risco que a semeadura nessa época apresenta no que diz respeito à ferrugem asiática da soja: a chamada "ponte verde", que pode existir em relação à fonte de inoculo da doença, que permanece viável na entre-safra. Segundo Embrapa (2005), fungos causadores de ferrugens são classificados como biotróficos, ou seja, necessitam do hospedeiro vivo para sobreviver e se multiplicar. Portanto, a sobrevivência de Phakopsora pachyrhizi, na entre-safra, tem ocorrido em cultivos de soja sob irrigação no inverno, o que pode vir a prejudicar a safra de soja de verão que sucede a da entresafra. No entanto, a qualidade fisiológica superior das sementes nessa época alternativa é uma das grandes vantagens.

Além da qualidade superior de sementes, o cultivo na entre-safra é uma excelente opção para a rotação de culturas, permitindo, ainda, a obtenção de uma segunda safra no mesmo ano, proporcionando cobertura vegetal do solo por maior período, propiciando o retorno de boa quantidade de resíduos vegetais após a colheita e reduzindo o tempo de armazenamento necessário entre a colheita e a próxima semeadura. O problema do déficit hídrico, comum nessa época em várias regiões, pode ser contornado por meio da irrigação suplementar, quando possível, evitando redução na produtividade (Lazarini, 1995).

Para a adoção dessa época alternativa de semeadura, é necessário o conhecimento do desempenho dos diversos materiais genéticos existentes, uma vez que no período de entre-safra, que compreende os meses de maio a agosto, há alterações no ciclo das cultivares, causadas por mudanças no fotoperíodo, com conseqüências principalmente sobre a produtividade.

Portanto, se torna necessária a seleção de genótipos com capacidade para produzir sementes em quantidade $\mathrm{e}$ qualidade nessas condições. Isto porque, segundo Rocha e Vello (1999), o desempenho de um genótipo é a ação conjunta dele, do ambiente e da interação entre esses fatores, sendo que essa interação reflete as diferenças na sensibilidade dos genótipos às variações ambientais, resultando em alterações em seu desempenho.

Para alguns estados existem, há algum tempo, recomendações de cultivares para semeadura fora dos meses de outubro e novembro, como o mês de fevereiro no Paraná (Ocepar/Embrapa, 1990), e final de abril e início de maio em Goiás, Tocantins e Distrito Federal (Embrapa, 1993).

A região de Selvíria, MS, apresenta potencial para cultivo da soja em entre-safra com a finalidade de produção de sementes com elevado potencial fisiológico, porque possui disponibilidade de água para irrigação e temperaturas amenas no inverno.

Sementes de diferentes cultivares, semeadas no mês de maio nesse mesmo local, apresentaram germinação e vigor, medido pelos testes de envelhecimento acelerado, índice de velocidade de germinação e condutividade elétrica, superiores às outras, cuja semeadura se deu em novembro (Lazarini et al., 2001). Assim, ensaios de competição com número maior de cultivares tornam-se importantes, pois permitirão uma seleção mais precisa de materiais com potencial para utilização nessa região. 
O objetivo do presente trabalho foi avaliar as características agronômicas, a produção e a qualidade fisiológica das sementes de cultivares de soja em semeadura de inverno, em Selvíria, MS.

\section{MATERIAL E MÉTODOS}

O trabalho foi conduzido na Fazenda Experimental da Universidade Estadual Paulista - UNESP, câmpus de Ilha Solteira, localizada no município de Selvíria, MS (20²2'S, $51^{\circ} 22^{\prime} \mathrm{W}$ e altitude média de $\left.335 \mathrm{~m}\right)$. O clima da região é do tipo Aw, segundo a classificação de Köeppen, com temperatura e precipitação pluvial média anual de $23,5^{\circ} \mathrm{C}$ e $1370 \mathrm{~mm}$, respectivamente (Hernandez et al., 1995). O solo da área experimental é considerado como LATOSSOLO VERMELHO Distrófico (Embrapa, 1999a). Na Tabela 1 encontram-se os resultados da análise química de uma amostra de solo da área experimental, retirada a 0-20 cm de profundidade, antes da instalação do experimento. $\mathrm{Na}$ adubação foram colocados $250 \mathrm{~kg} \mathrm{ha}^{-1}$ da fórmula 4-3010.

Foram utilizadas sementes das cultivares FT-2000, FTInaê, Embrapa 20 (Doko RC), CAC-1, IAC-17, IAC-18, IAC-19, IAC-8-2, FT-101, FT-109, MT/BR 45 (Paiaguás), MT/BR 50 (Parecis), MT/BR 52 (Curió), MT/BR 53 (Tucano), MT/BR 47 (Canário), MT/BR 49 (Pioneira), BRSMT Uirapuru, BR Emgopa 314 (Garça Branca), MG/ BR 46 (Conquista), FT-Estrela, FT-Cometa, Dourados, JAB-11, BR 9 (Savana), FT-Abyara, Embrapa 30 (Vale do Rio Doce), Embrapa 9 (Bays), Embrapa 31 (Mina), IAC16, IAS 5, EMGOPA-304 e IAC-Foscarin 31, semeadas mecanicamente, em espaçamento de $0,5 \mathrm{~m}$ entre linhas, com densidade de semeadura visando obter população de
400.000 plantas por hectare. A semeadura ocorreu em 5/6, e a colheita, de 14/9 a 14/10/1998. O tratamento de sementes, controle de plantas daninhas, pragas e doenças, foram feitos segundo recomendações da Embrapa (1999b).

Foram avaliadas a altura de plantas e de inserção das primeiras vagens, por meio de cinco plantas coletadas aleatoriamente na parcela.

Para produtividade, coletaram-se as plantas contidas em duas linhas com dois metros de comprimento, aleatoriamente em cada parcela; essas plantas foram secadas ao sol e trilhadas mecanicamente. Adicionalmente, determinou-se o teor de água das sementes, pelo método da estufa (Brasil, 1992), para correção do peso obtido a 13\% (base úmida).

A viabilidade foi determinada pelo teste de germinação segundo Brasil (1992) e o vigor, pelos testes de envelhecimento acelerado e de condutividade elétrica (Vieira e Carvalho, 1994).

Para a análise estatística dos resultados, usou-se o delineamento em blocos ao acaso. As médias foram comparadas usando-se o teste de Tukey, a 5\% de probabilidade (Banzato e Kronka, 1995).

\section{RESULTADOS E DISCUSSÃO}

Nenhuma cultivar apresentou altura mínima de planta considerada adequada para a colheita mecânica, que segundo Bonetti, (1983), deve ser superior a $65 \mathrm{~cm}$. Do mesmo modo, apenas uma das sete cultivares estudadas por Crusciol et al. (2002), na mesma região e época, superou essa altura. Valores entre 50 e $60 \mathrm{~cm}$, foram alcançados pelas cultivares IAC-19, IAC-8-2, FT-109, Paiaguás, Pioneira, Conquista, Savana e Mina.

TABELA 1. Análise química de amostra de solo $(0-20 \mathrm{~cm}$ de profundidade) da área experimental.

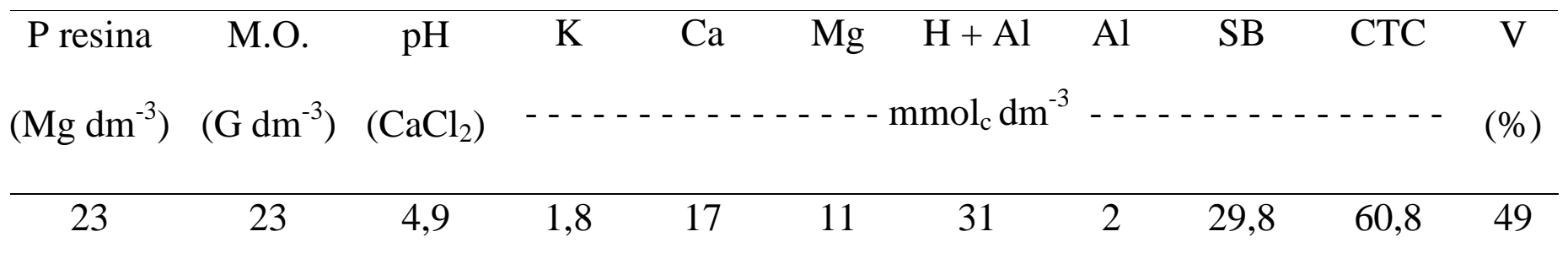


Quanto à altura de inserção da primeira vagem, apenas as cultivares FT 2000, FT-Inaê, Doko RC, IAC-17, IAC18, FT-101, FT-Cometa, Dourados, FT-Abyara, Estrela e Vale do Rio Doce não alcançaram o valor mínimo de 10 cm, considerado desejável para a colheita mecânica (Bonetti, 1983). No trabalho de Crusciol et al. (2002) na mesma região, apenas três cultivares não se enquadraram no valor mínimo.

Trabalhando com 12 cultivares de soja, em duas épocas de semeadura (novembro e março), Lazarini et al. (2001) observaram redução na altura de plantas de todos os materiais semeados em março, em relação ao período convencional. Medina et al. (1997b) também observaram menor altura de plantas e de inserção das primeiras vagens das plantas semeadas em março.

Em períodos de dias curtos, é possível que haja encurtamento do ciclo das cultivares, em função da antecipação do florescimento, o que reduz a altura de plantas, de inserção das primeiras vagens, com número conseqüentemente menor de entrenós, alterações estas relatadas por Tragnago \& Bonetti (1984) e Board \& Settimi (1986).

Adaptações nos equipamentos podem ser feitas visando à colheita de plantas com altura inferior a $65 \mathrm{~cm}$, mas com a primeira vagem, a pelo menos, $10 \mathrm{~cm}$ do solo, reduzindo desse modo as perdas motivadas por vagens não-colhidas em função da baixa altura de inserção (Lazarini, 1995).

Os resultados da avaliação da produtividade, da germinação, do envelhecimento acelerado e da condutividade elétrica são apresentados na Tabela 2. Pelos dados de produtividade, pode-se verificar que houve grande variação entre os valores obtidos pelas diversas cultivares. Todas apresentaram valores abaixo da média verificada no período convencional no Estado de Mato Grosso do Sul, que foi de $2600 \mathrm{~kg} \mathrm{ha}^{-1} \mathrm{em} 1999$ (AGRIANUAL, 2000). O encurtamento do ciclo e a baixa altura de plantas, devido ao efeito fotoperiódico, levam à formação de menor número de entrenós, reduzindo a quantidade de sementes por planta, com conseqüências sobre o rendimento de sementes.

Tomando-se por referência a maior produtividade, alcançada pela cultivar FT-2000 (1764 kg ha-1), devem ser descartadas as cultivares Emgopa-304, Dourados, FTCometa, IAC-Foscarin, Savana, FT-Estrela, FT-Abyara, Mina, FT-101, IAC-17 e Vale do Rio Doce, que diferiram estatisticamente da primeira, apresentando produtividades abaixo de $1290 \mathrm{~kg}$. ha ${ }^{-1}$ de sementes.

Peixoto et al. (2000), trabalhando com três cultivares, épocas de semeadura e densidades de plantas, encontraram para a densidade de 400.000 plantas ha $^{-1}$, a mesma utilizada na presente pesquisa, resultados de produtividade de grãos para a época de safrinha muito abaixo dos da época convencional e tardia. Os autores enfatizam que a época de safrinha pode ser uma opção para a produção de sementes, mesmo com produtividade inferior. Esses resultados corroboram as observações de Nakagawa et al. (1983), Tragnago e Bonetti (1984) e Medina et al. (1997a).

A produtividade de $47 \%$ das 12 cultivares avaliadas por Lazarini et al., (2001) na região de Selvíria, MS, foi inferior na semeadura de inverno, em relação à da época convencional. Em trabalho anterior, no entanto, Lazarini et al. (1992) constataram que, embora as sete cultivares estudadas não apresentassem características desejáveis para a colheita mecânica, a produtividade de algumas chegou a $3000 \mathrm{~kg} \mathrm{ha}^{-1}$. Sendo o rendimento da cultura grandemente influenciado pelas condições ambientais, é de se esperar resultados diferenciados nesse parâmetro, conforme verificou esse autor nessas duas pesquisas, pois as condições ambientais variam de um ano agrícola para outro, variando também a interação de cada genótipo com o ambiente. Isso ficou comprovado por Rocha e Vello (1999).

Assim, a produtividade das plantas na semeadura em época de safrinha poderá até se igualar à da época convencional, caso a temperatura e a umidade não limitem o desenvolvimento da cultura, pois, segundo Chaves et al. (1989), o ambiente pode favorecer a expressão do potencial dos genótipos, maximizando o rendimento.

Os testes para avaliação da qualidade fisiológica das sementes, das diferentes cultivares, também mostraram que estas diferiram significativamente entre si nesses aspectos.

Quanto ao teste de germinação, aproximadamente 66\% das cultivares atingiram o valor mínimo preconizado para a semeadura no Estado de Mato Grosso do Sul, que é de 80\% (Embrapa, 1993). Entretanto, nem todas tiveram desempenho semelhante nas outras avaliações.

No teste de envelhecimento acelerado, observou-se bom desempenho das sementes, não se verificando redução na porcentagem de plântulas normais em função das condições 
impostas pelo teste. Esse resultado indica a possibilidade de ter ocorrido algum dano de embebição ou algum efeito prejudicial causado por fungos de campo, como os dos gêneros Phomopsis e/ou Fusarium, que podem afetar os resultados do teste de germinação em rolo de papel; assim, os resultados de envelhecimento acelerado podem ser maiores (Henning e França Neto, 1980; França Neto et al., 1998).

Por esse teste, apenas as sementes das cultivares IAC19, FT-101, JAB-11, Savana e Vale do Rio Doce foram consideradas inadequadas para a semeadura, porque, segundo Egli e TeKrony (1995), sementes com germinação inferior a $80 \%$ nesse teste têm pouca possibilidade de conseguir emergência adequada de plântulas em campo, sob condições inadequadas de ambiente.

No teste de condutividade elétrica, sementes com valores até $60-70 \mathrm{mS} \mathrm{cm}^{-1} \mathrm{~g}^{-1}$ tem sido consideradas como de alto vigor, enquanto $70-80 \mathrm{mS} \mathrm{cm} \mathrm{cm}^{-1} \mathrm{~g}^{-1}$ já são valores com tendência para médio vigor (AOSA, 2002). Por essa definição, pode-se inferir que metade das cultivares mostraram-se inaptas para a semeadura.

O desempenho de todas as cultivares no teste de envelhecimento acelerado foi superior ao encontrado por Lazarini et al. (2001) em época semelhante, com exceção das cultivares JAB-11, Savana e Vale do Rio Doce, e desempenho inferior para todas as cultivares no teste de condutividade elétrica, uma vez que apresentaram valores mais elevados de condutividade elétrica na solução de embebição.

As cultivares Doko RC, FT-Inaê, IAC-18 e FT-2000 não se mostraram como opção interessante para semeadura porque, mesmo com excelentes valores de produtividade $\mathrm{e}$ potencial fisiológico (Tabela 2), não atingiram altura mínima $(10 \mathrm{~cm})$, recomendada para colheita mecânica sem perdas consideráveis. As cultivares FT-Cometa, IAC-Foscarin e IAC-17, mesmo com valores elevados de germinação e vigor, não devem ser recomendadas, porque suas produtividades foram estatisticamente inferiores à da que obteve o melhor resultado (FT-2000). As cultivares JAB11, Uirapuru, Curió, Canário e Tucano apresentaram boa produtividade, mas os dados de viabilidade e vigor não se mostraram consistentes, ou seja, elas apresentaram bom desempenho nos testes padrão de germinação e de envelhecimento acelerado, o que não ocorreu no teste de condutividade elétrica, indicando algum aspecto de deterioração nas sementes.

Por outro lado, a cultivar IAS-5 mostrou resultados positivos em produtividade e nos testes de envelhecimento acelerado e de condutividade elétrica, mas valores abaixo do esperado no teste de germinação. IAC 8-2 e IAC-19 só se destacaram em produtividade, ao passo que Conquista e FT-109 destacou-se apenas em produtividade e nos resultados do envelhecimento acelerado. Já Emgopa 304, Dourados, Savana, FT-Estrela, FT-Abyara, Mina, FT-101 e Vale do Rio Doce tiveram produtividade inferior estatisticamente à de maior valor (FT-2000), embora algumas tenham sido consideradas de viabilidade e vigor satisfatórios.

De um modo geral, as cultivares que mais se destacaram e mostraram aptidão para semeadura no inverno, nessa região, foram Parecis, Bays, CAC-1, Garça Branca, Paiaguás, Pioneira e IAC-16, com produtividades que variaram de aproximadamente 1.300 a $1.700 \mathrm{~kg} \mathrm{ha}^{-1}$ e germinação e vigor de $87 \%$ a $100 \%$. As cultivares Bays e Parecis destacaram-se, mesmo com condutividade elétrica superior em $80 \%$, porque esse valor não diferiu estatisticamente do menor valor $\left(55,0 \mathrm{mS} \mathrm{cm}^{-1} \mathrm{~g}^{-1}\right)$, que foi da cultivar Garça Branca. No entanto, tem que se considerar o efeito do genótipo com relação aos valores de condutividade elétrica, ou seja, cultivares com valores semelhantes de germinação podem apresentar resultados distintos de condutividade elétrica, fatos também verificados na literatura, conforme observado por Bedford (1974) em ervilha, Tao (1978) e Bruggink et al. (1991) em milho, e Panobianco \& Vieira (1996) em soja.

Embora a produtividade alcançada seja considerada baixa, ela pode ser compensada pelo valor agregado às sementes, que serão de elevada qualidade, possibilitando ao agricultor incrementar seus rendimentos. Ainda, de acordo com Lazarini et al. (2001), outra vantagem nesse sentido, é que, efetuando-se a semeadura entre maio e junho, a colheita será realizada a partir de setembro e outubro, fazendo com que, caso o produtor queira utilizar essas sementes para a semeadura de novembro, época convencional, elas ficarão armazenadas apenas dois meses. 
TABELA 2. Produtividade, germinação, envelhecimento acelerado e condutividade elétrica de sementes das diferentes cultivares de soja, cultivadas no inverno.

\begin{tabular}{|c|c|c|c|c|}
\hline Cultivares & Produtividade & Germinação & Env. acelerado & Condutiv. elétrica \\
\hline & $\left(\mathrm{kg} \mathrm{ha}^{-1}\right)$ & \multicolumn{2}{|c|}{$\begin{array}{l}-\ldots \ldots \\
\end{array}$} & $\left(\mu \mathrm{S} \mathrm{cm}^{-1} \mathrm{~g}^{-1}\right)$ \\
\hline FT-2000 & $1764 \mathrm{a}$ & 85 abcde & 87 abcdef & 74,8 ghijkl \\
\hline Parecis & $1726 a b$ & $95 \mathrm{ab}$ & 94 abcd & 83,9 efghijkl \\
\hline JAB-11 & $1720 \mathrm{ab}$ & 77 bcdefg & $73 \mathrm{~g}$ & $134,6 \mathrm{ab}$ \\
\hline CAC-1 & $1703 \mathrm{abc}$ & 82 abcde & $95 \mathrm{abc}$ & $65,3 \mathrm{ijkl}$ \\
\hline Pioneira & $1700 \mathrm{abc}$ & $87 \mathrm{abcd}$ & 92 abcde & 73,0 hijkl \\
\hline IAC-18 & $1687 \mathrm{abc}$ & 91 abcd & $95 \mathrm{abc}$ & 71,3 hijkl \\
\hline Uirapuru & $1677 \mathrm{abcd}$ & 80 abcde & 90 abcdef & 102,0 cdefgh \\
\hline Garça Branca & 1610 abcde & $94 \mathrm{abc}$ & $98 \mathrm{ab}$ & 55,01 \\
\hline Doko RC & 1603 abcde & 92 abcd & 94 abcd & $64,2 \mathrm{ijkl}$ \\
\hline IAS-5 & 1543 abcdef & 78 abcdef & 92 abcde & $68,2 \mathrm{ijkl}$ \\
\hline IAC $8-2$ & 1537 abcdef & 60 fghij & 80 efg & 105,1 bcdefg \\
\hline Curió & 1517 abcdef & 80 abcde & 81 defg & 113,9 bcdef \\
\hline Paiaguás & 1503 abcdef & 92 abcd & $96 \mathrm{abc}$ & 73,0 hijkl \\
\hline Conquista & 1500 abcdef & 73 defghi & 84 cdefg & $116,8 \mathrm{bcd}$ \\
\hline IAC-19 & 1477 abcdef & 58 ghij & $79 \mathrm{fg}$ & 155,3 a \\
\hline Bays & 1457 abcdefg & 89 abcd & $95 \mathrm{abc}$ & 80,5 ghijkl \\
\hline Canário & 1400 abcdefgh & 88 abcd & 91 abcdef & 94,8 defghi \\
\hline FT-109 & 1387 abcdefgh & 76 cdefg & 85 bcdefg & 91,9 defghi \\
\hline FT-Inaê & 1383 abcdefgh & $87 \mathrm{abcd}$ & $95 \mathrm{abc}$ & $59,8 \mathrm{kl}$ \\
\hline Tucano & 1320 abcdefghi & $90 \mathrm{abcd}$ & 89 abcdef & 92,3 defghi \\
\hline IAC-16 & 1290 abcdefghi & $96 \mathrm{a}$ & $95 \mathrm{abc}$ & 76,12 ghijkl \\
\hline Emgopa 304 & 1277 bcdefghi & 74 defgh & $96 \mathrm{abc}$ & 58,31 \\
\hline Dourados & 1273 bcdefghi & 80 abcde & $95 \mathrm{abc}$ & 75,7 ghijkl \\
\hline FT-Cometa & 1237 cdefghi & $97 \mathrm{a}$ & $100 \mathrm{a}$ & $59,9 \mathrm{kl}$ \\
\hline IAC-Foscarin & 1203 defghi & 96 a & $96 a b c$ & $69,9 \mathrm{ijkl}$ \\
\hline Savana & 1183 efghi & $46 \mathrm{j}$ & $72 \mathrm{~g}$ & $129,7 \mathrm{abc}$ \\
\hline FT-Estrela & 1173 efghi & 86 abcde & 99 a & $61,0 \mathrm{jkl}$ \\
\hline FT-Abyara & 1110 fghi & 68 efghi & 91 abcdef & 90,3 defghijk \\
\hline Mina & 993 ghi & 76 cdefg & 81 efg & 114,1 bcde \\
\hline FT-101 & 970 hi & $55 \mathrm{ij}$ & $78 \mathrm{fg}$ & 82,9 fghijkl \\
\hline IAC-17 & $880 \mathrm{i}$ & $97 \mathrm{a}$ & $97 \mathrm{abc}$ & 58,61 \\
\hline Vale do Rio Doce & $870 \mathrm{i}$ & 57 hij & $24 \mathrm{~h}$ & $148,5 \mathrm{a}$ \\
\hline C.V. $(\%)$ & 13,9 & 9,0 & 5,0 & 13,0 \\
\hline
\end{tabular}

Médias seguidas por letras distintas na coluna diferem entre si $(\mathrm{P}<0,05)$ pelo Teste de Tukey. 


\section{CONCLUSÕES}

Para semeadura de inverno na região de Selvíria, MS, deve-se atentar para a escolha de cultivares mais adaptadas às condições climáticas da região, destacando-se as cultivares Parecis, Bays, CAC-1, Garça Branca, Paiaguás, Pioneira e IAC-16, em relação aos valores de produtividade e vigor.

A região pode ser considerada como promissora para produção de sementes de elevada qualidade fisiológica, em semeadura de inverno, alertando-se para o fato de que a semeadura com suplementação hídrica, e, principalmente, escolha de cultivar adaptada.

\section{REFERÊNCIAS}

AGRIANUAL 2000: anuário da agropecuária brasileira. São Paulo: FNP Consultoria \& Comércio, 1999. p.499.

AOSA-ASSOCIATION OF OFFICIAL SEED ANALYSTS. Seed vigor testing handbook. Lincoln: AOSA, 2002. 105p. (Contribuition, 32)

BANZATO, D.A.; KRONKA, S.N. Experimentação agrícola. 3.ed. Jaboticabal: FUNEP, 1995. 247p.

BEDFORD, L.V. Conductivity tests in commercial and hand harvested seeds of pea cultivars and their relation to field establishment. Seed Science and Technology, Zürich, v.2, n.3, p.323-335, 1974.

BOARD, J.E., SETTIMI, R. Photoperiod effect before and after flowering on branch development in determinate soybean. Agronomy journal, Madison, v.78, p.905-1002, 1986.

BONETTI, L.P. Cultivares e seu melhoramento genético. In: VERNETTI,F.J.(Coord.). Soja: genética e melhoramento. Campinas: Fundação Cargill, 1983. p.741-794.

BRACCINI, A.L.E.; MOTTA, I.S.; SCAPIM, C.A.; BRACCINI, M.C.L.; ÁVILA, M.R.; SCHUAB, S.R.P. Semeadura da soja no período da safrinha: potencial fisiológico e sanidade das sementes. Revista Brasileira de Sementes, Brasília, v.25, n.1, p.76-86, 2003.

BRASIL. Ministério da agricultura e Reforma Agrária. Secretaria Nacional de Defesa Agropecuária. Regras para análise de sementes. Brasília, 1992.365p.

BRUGGINK, H.; KRAAK, H.L.; DIJEMA, M.H.G.E.; BEKENDAM, J. Some factors influencing eletrolyte leakage from maize (Zea mays L.) Kernels. Seed Science Research, London, v.1, n.1, p.1520, 1991.
CHAVES, L.J.; VENCOVSKY, R.; GERALDI, I.O. Modelo não linear aplicado ao estudo da interação genótipo $\mathrm{x}$ ambiente em milho. Pesquisa Agropecuária Brasileira, Brasília, v.24, n.2, p.259-269, 1989.

CRUSCIOL, C.A.C.; LAZARINI, E.; BUZO, C.L.; SÁ, M.E. Produção e qualidade fisiológica de sementes de soja avaliadas na semeadura de inverno. Scientia Agricola, Piracicaba, v.59, n.1, p.79-86, 2002.

EGLI, D.B.; TEKRONY, D.M. Soybean seed germination, vigor and field emergence. Seed Science and Technology, Zürich, v.23, n.3, p.595-607, 1995.

Empresa Brasileira de Pesquisa Agropecuária. Serviço de produção de sementes básicas. Padrões estaduais de sementes. Brasília, 1993. p.35-37.

Empresa Brasileira de Pesquisa Agropecuária. Centro Nacional de Pesquisa de Solo. Sistema brasileiro de classificação de solos. Rio de Janeiro, 1999a. 412p.

Empresa Brasileira de Pesquisa Agropecuária. Centro Nacional de Pesquisa de Soja. Recomendações para a cultura da soja na região central do Brasil - 1999/2000. Londrina, 1999b. 226p. (Embrapa Soja, Documentos, 132).

Embrapa Brasileira de Pesquisa Agropecuária. Tecnologias de produção de soja - Paraná 2006. Londrina, 2005. 208p. (Sistemas de Produção/Embrapa Soja, ISSN 1677-8499; n.8).

FRANÇA NETO, J.B.; KRZYZANOWSKI, F.C.; HENNING, A.A.; COSTA, N.P. Suscetibilidade das principais cultivares de soja utilizadas no Brasil ao dano de embebição no teste de germinação. Londrina: EMBRAPA-CNPSo, 1998. 10p. (EMBRAPA-CNPSo, Comunicado Técnico, 60).

HENNING, A.A.; FRANÇA NETO, J.B. Problemas na avaliação da germinação de sementes de soja com alta incidência de Phomopsis sp.. Revista Brasileira de Sementes, Brasília, v.2, n.3, p.922, 1980 .

HERNANDEZ, F.B.T., LEMOS FILHO, M.A.F., BUZZETTI, S. Software Hidrisa e o balanço hídrico de Illha Solteira. Ilha Solteira: UNESP/FEIS/Área de Hidráulica e Irrigação, 1995. 45p. (UNESP/ FEIS/Área de Hidráulica e Irrigação. Série Irrigação, 1).

LAZARINI, E. Avaliação das características agronômicas e análises nutricionais de genótipos de soja semeados em diferentes épocas, em Jaboticabal, SP. 1995. 197p. Tese (Doutorado em Fitotecnia) - Universidade Estadual Paulista UNESP, Jaboticabal, 1995.

LAZARINI, E.; SÁ, M.E.; BUZO, C.L.; CRUSCIOL, C.A.C. Avaliação dos caracteres produtivos e produção de grãos de soja, semeada no período de inverno e de verão, na região de Selvíria-MS. In: CONGRESSO DE INICIAÇÃOCIENTÍFICA, 4., 1992, Araçatuba. Anais... Araçatuba: UNESP, 1992. p.12-13. 
LAZARINI, E.; SÁ, M.E.; CARVALHO, G.R. Efeito da época de semeadura e de colheita na produção e qualidade fisiológica de sementes de diferentes variedades de soja. In: CONGRESSO BRASILEIRO DE SEMENTES, 12., 2001, Londrina. Anais... Londrina: Informativo ABRATES, 2001. v.11, n.2, p.95.

MEDINA, P.F.; RAZERA, L.F.; MARCOS FILHO, J.; BORTOLETTO, N. Produção de sementes de cultivares precoces de soja em duas épocas e dois locais paulistas: I. Características agronômicas e produtividade. Bragantia, Campinas, v.56, n.2, p.291-303, 1997a.

MEDINA, P.F.; RAZERA, L.F.; MARCOS FILHO, J.; BORTOLETTO, N. Produção de sementes de cultivares precoces de soja em duas épocas e dois locais paulistas: II. Qualidade fisiológica. Bragantia, Campinas, v.56, n.2, p.305-315, 1997 b.

NAKAGAWA, J.; ROSOLEM, C.A.; MACHADO, J.R. Épocas de semeadura de soja: I. Efeito na produção de grãos e nos componentes de produção. Revista Brasileira de Sementes, Brasília, v.18, n.11, p.1187-1198, 1983.

NAKAGAWA, J.; ROSOLEM, C.A.; MACHADO, J.R. Efeito da época de semeadura na qualidade de sementes de três cultivares de soja, em Botucatu-SP. Revista Brasileira de Sementes, Brasília, v.6, n.1, p.25-38, 1984a.

NAKAGAWA, J.; ROSOLEM, C.A.; MACHADO, J.R. Desempenho de sementes de soja originárias de culturas estabelecidas em diferentes épocas. Revista Brasileira de Sementes, Brasília, v.6, n.3, p.61-76, 1984 b.

OCEPAR/EMBRAPA. Recomendações técnicas para a cultura da soja no Paraná (1990/1991). Cascavel: OCEPAR/EMBRAPA/ CNPSo, 1990. 100p. (Boletim Técnico, 27)
PANOBIANCO, M.; VIEIRA, R.D. Electrical conductivity of soybean soaked seeds. I. Effect of genotype. Pesquisa Agropecuária Brasileira, Brasília, v.31, n.9, p.621-627, 1996.

PAOLINELLI, G.P.; TANAKA, M.A.S.; REZENDE, A.M. Influência da época de semeadura sobre a qualidade de sementes de soja. Revista Brasileira de Sementes, Brasília, v.6, n.1, p.39-50, 1984.

PEIXOTO, C.P.; CÂMARA, G.M.S.; MARTINS, M.C.; MARCHIORI, L.F.S.; GUERSONI, R.A.; MARTIAZZI, P. Épocas de semeadura e densidades de plantas de soja: I. Componentes da produção e rendimento de grãos. Scientia Agrícola, Piracicaba, v.57, n.1, p.89-96, 2000.

ROCHA, M.M.; VELLO, N.A. Interação genótipos e locais para rendimento de grãos de linhagens de soja com diferentes ciclos de maturação. Bragantia, Campinas, v.58, n.1, p.69-81, 1999.

TAO, K.L.J. Factors causing variations in the conductivity test for soybean seeds. Journal of Seed Technology, Springfield, v.3, n.1, p.10-18, 1978.

TEKRONY,D.M.; EGLI, D.B.; BALLES, J.; TOMES, L.; STUCKEY, R.E. Effect of date of harvest maturity on soybean seed quality and Phomopsis sp. seed infection. Crop Science, Madison, v.24, n.1, p.189-193, 1984.

TRAGNAGO, J.L.; BONETTI, L.P. Diferentes épocas de semeadura no rendimento e outras características de alguns cultivares de soja no Rio Grande do Sul. In: SEMINÁRIO NACIONAL DE PESQUISA DE SOJA, 3., 1984, Brasília. Anais... Londrina: EMBRAPA/CNPSo, 1984. p.57-69.

VIEIRA, R.D.; CARVALHO, N.M. Testes de vigor em sementes. Jaboticabal: FUNEP, 1994. 164p. 